\title{
Mechanical System Design and Analysis of Three Dimensional Scanning Probe Based on Flexible Parallel Mechanism
}

\author{
Beibei Wu${ }^{1}$, a, Hongxi Wang ${ }^{1}$, Yaxiao Wang ${ }^{1}$, Weiwei $\mathrm{Li}^{1}$ \\ ${ }^{1}$ Xi'an Technological University \\ awubei123456@foxmail.com
}

Keywords: Canning probe; Parallel mechanism; Decoupling

\begin{abstract}
Mechanical coupling is one of the key factors that cause scanning probe mechanical error. Minimizing or eliminating the mechanical coupling error can Optimize structural design of the probe. A three-dimensional scanning probe is designed with three-brach flexibility parallel mechanism, whose guiding stiffness based on the energy method is derived. Mechanical coupling error is simulated and analyzed with finite element method in the paper. The result shows that three-branch parallel scanning probe reduces the mechanical coupling error to about $20 \%$ of the error of serial mechanism probe.
\end{abstract}

\section{Introduction}

With the rapid development of science and technology, coordinate measuring machine (CMM), a typical representative of modern measuring technology, has been widely used in industry. The probe is an important component of CMM and its development affects the performance of CMM. Only when the precise measuring probe provides the new measuring principle and the new measuring accuracy for CMM, can precision and efficiency of the CMM get further development ${ }^{[1]}$.

Touch scanning probe, a kind of device which is often used in precise measurement, has many advantages such as high precision, good repeatability, no pre stroke error and so on. Some famous companies such as the United Kingdom Renishaw, Germany's Klingberg, Leitz and Zeiss Carl, Switzerland's Mecartex and the United States EMD and so on, have been in a leading position in studying the scanning probe. The measuring probe that German Mauser-WerkeOberndorf GmbH developed, whose guiding mechanism adopts three-layer reed, is a device with the typically serial structure $^{[2]}$ and has the advantages of simple structure. But moving parts of each measuring axis have different quality, which leads to the different dynamic characteristics of each directions. A parallel scanning probe, German FritzErtl Schweinfurt designs in patent U.S.Pat No.5029398 ${ }^{[3]}$, whose guiding mechanism is composed of parallel spring mechanism in flexible hinge mechanism, but it is difficult to manufacture and assemble for the mechanism. A compound serial-parallel probe ${ }^{[4]}$, Germany Klingelnberg designs in a patent, compensates measuring error with the two-dimensional hinge bar, but it is difficult to manufacture for its two-dimensional trapezoidal hinge bar. TRAX, a scanning probe system developed German Leitz ${ }^{[5]}$, avoids the cosine error and eliminates the mechanical zero error, which leads to the high accuracy. But it is difficult to promote practical use because the motion of each axis is realized by sliding friction.

At present, the common scanning probe especially the traditional series probe has the mechanical coupling phenomenon in scanning measurement. It has larger mechanical coupling error and lower measuring precision. Therefore, it is the focus for our research to design a scanning probe with small coupling or even no coupling.

\section{Structure and Principle of Scanning Probe}

At present, the common scanning probe with serial-parallel form still exists mechanical coupling error. Therefore, it is the focus of designing a decoupled scanning probe based on the parallel principle, minimizing the mechanical coupling error. In this project, the parallel ${ }^{[6-9]}$ guiding mechanism is composed of three identical integrated hinges. Displacement detection method adopts 
inductive displacement transducer. The variation of the micro displacement about the guiding mechanism leads to the variation of inductance. Fig. 1(a) and Fig. 1(b) show the schematic and 3D organization chart of the scanning probe.

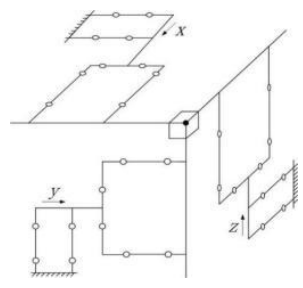

Figure 1(a). The schematic

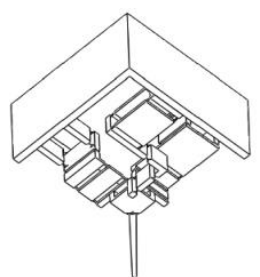

Figure 1(b). Three-dimensiona organization chart

The characteristic of three-branch scanning probe is how to design the guiding chain. Fig. 2 shows 3D drawing of three-branch scanning probe, including the hinge in chains (1), (2), (3) can carry on $3 \mathrm{D}$ space orthogonal motion ${ }^{[10]},(4)$ and (5) show respectively active plate and the fixed plate of the scanning probe, (6) and (7) respectively show movable core and fixed core of the inductance sensor. When the probe is scanning measurement, active plate (4) moving core (6) produce translation, leading to the variation of inductance and detecting the displacement of moving plate (4). In general, translational motion of the active plate (4) produces a vertical motion, whose the displacement that is called coupling error cannot be detected through the sensor installing on active plate (4) and fixed plate (5). However, the hinge (3) can produce a protrusive bending deformation that amounts of the coupling error, which drives active plate on another chain moving and greatly minimizes the mechanical coupling error.

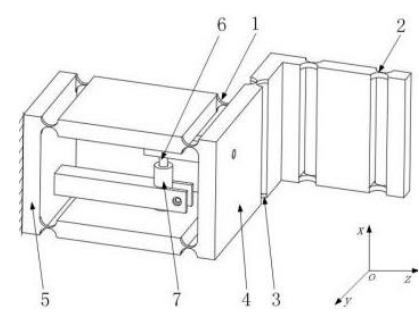

Figure 2. Branched 3D graph of scanning probe

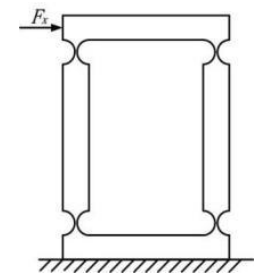

Figure 3. Double straight round flexure hinge

\section{Stiffness Calculation of the Probe}

Theoretical Calculation of the Probe'S Stiffness. According to the structure principle of the scanning probe in the design, the structure size of three guiding chains is identical. A local coordinate system oxyz is established with any chains. The stiffness of single chain show $k_{i x}, k_{i y}, k_{i z}$ ( $i$ is the number of chain, for $i=1,2,3$ ). The stiffness of single chain under the action of measuring force $F_{x}$ is $k_{x}$, and the stiffness of the under the action of the force $F_{y}$ is $k_{y}$, and the stiffness of the under the action of the force $F_{z}$ is $k_{z}$. The direction of the measuring force $F_{x}$ is the same as the translational direction of the hinge (1) in the chain (as shown in Fig. 2), The direction of the measuring force $F_{y}$ is the same as the translational direction of the hinge (2) in the chain, The direction of the measuring force $F_{z}$ is the same as the translational direction of the hinge (3) in the chain. The elastic parallelogram guiding mechanism can be simplified as double straight round flexure hinge, referring to Fig. 3, then the stiffness of the hinge (1) is calculated according to the method of double straight round flexible hinge, the stiffness of the hinges (2) and (3) are calculated according to the method of energy conservation.

According to how to design flexure hinges ${ }^{[1]}$, when the thickness of the hinge is much smaller than the radius $\mathrm{R}$ and the width $B_{1}$, the bending stiffness can be simplified as: 


$$
K_{\alpha}=\frac{2 E B_{i} t^{5 / 2}}{9 \pi R^{1 / 2}}
$$

Where, $E$ isthe elastic modulus, $B_{i}$ is width of the hinge, $t$ is thickness of the hinge, $R$ is radius of the hinge.

When the measuring force acting on the chain is $F_{x}$, as shown in Fig. 3, the hinge (1) produces deformation.

The span of the hinge (1) is $l$, then the translational stiffness $K_{x}$ of the chain under the action of the measuring force $F_{x}$ are calculated as follows:

$$
k_{x}=4 \frac{\mathrm{K}_{\alpha}}{l^{2}}=\frac{8 \mathrm{~EB}_{1} t^{5 / 2}}{9 \pi R^{1 / 2} l^{2}}
$$

When the measuring force acting on the chain is $F_{y}$, as shown in Fig. 4 (a), the hinge (2) and (3) produce deformation.

The translational stiffness of the hinge (2) and (3) based on the energy conservation are counted, the elastic energy of the guiding chain is counted that the work that $\Delta y$ displacement does under the action of measuring force $F_{y}$ translates. Fig. 4 (b) shows the deformation of the chain under the action of measuring force of $F_{y}$, among a, b, c are the span of the hinge, then rotating angles $\partial_{i}$ that each hinges generate show:

$$
\begin{aligned}
& \alpha_{1}=\left|\phi_{2}-\phi_{1}\right|, \alpha_{2}=\left|\phi_{4}-\phi_{3}\right|, \alpha_{3}=\left|\phi_{6}-\phi_{5}\right| \\
& \cos \phi_{1}=\frac{\left[(a+b-\Delta x)^{2}+c^{2}\right]+(a-\Delta x)^{2}-\left(b^{2}+c^{2}\right)}{2(a-\Delta x) \sqrt{(a+b-\Delta x)^{2}+c^{2}}} \\
& \cos \phi_{2}=\frac{\left[(a+b-\Delta x)+^{2} c+a^{2}-b^{2}+c^{2}\right)}{a \sqrt{(a+b-\Delta x)^{2}+c^{2}}} \\
& \cos \phi_{3}=\frac{[(a+b-\Delta x)+c]^{2}+\left(b^{2}+c^{2}\right)-(a-\Delta x)^{2}}{2 \sqrt{(a+b-\Delta x)+2} \sqrt[2]{b^{2}+c^{2}}} \\
& \cos \phi_{4}=\frac{\left[(a+b-\Delta x)+^{2} c+b^{2}+c^{2}\right)-a^{2}}{\sqrt[2]{(a+b-\Delta x)+2} \sqrt[2]{b^{2}+c^{2}}} \\
& \cos \phi_{5}=\frac{(a-\Delta x)^{2}+\left(b^{2}+c^{2}\right)-\left[(a+b-\Delta x)^{2}+c^{2}\right]}{2(a-\Delta x) \sqrt{b^{2}+c^{2}}} \\
& \cos \phi_{6}=\frac{a^{2}+\left(b^{2}+c^{2}\right)-\left[(a+b-\Delta x)^{2}+c^{2}\right]}{2 a \sqrt{b^{2}+c^{2}}}
\end{aligned}
$$

From conservation of energy can be determined:

$$
\begin{aligned}
& \frac{1}{2}{ }_{k} k \Delta^{2} y=\sum_{i=1}^{3} W_{i}=\sum_{i=1}^{3} \frac{1}{2} K_{\alpha_{i}} \alpha_{i}^{2} \\
& K_{\alpha_{1}}=K_{\alpha_{3}}=\frac{2 E B_{2} t^{5 / 2}}{9 \pi R^{1 / 2}}, K_{\alpha_{2}}=\frac{2 E B_{1} t^{5 / 2}}{9 \pi R^{1 / 2}}
\end{aligned}
$$

The translational stiffness $k_{y}$ of the chain under the action of measuring force $F_{y}$ is calculated as follows:

$$
k_{y}=K_{\alpha 1}\left(\alpha_{1} / \Delta y\right)^{2}+K_{\alpha 2}\left(\alpha_{2} / \Delta y\right)^{2}+K_{\alpha 3}\left(\alpha_{3} / \Delta y\right)^{2}
$$

When the measuring force acting on the chain is $F_{z}$, the hinge (2) and (3) are deformed, as shown in Fig. 5.

Similarly, the translational stiffness $k_{y}$ of the chain under the action of measuring force $F_{y}$ is calculated as follows: 


$$
\begin{aligned}
& \beta_{1}=\left|\theta_{2}-\theta_{1}\right|, \beta_{2}=\left|\theta_{4}-\theta_{3}\right|, \beta_{3}=\left|\theta_{6}-\theta_{5}\right| \\
& k_{z}=K_{\beta 1}\left(\beta_{1} / \Delta z\right)^{2}+K_{\beta 2}\left(\beta_{2} / \Delta z\right)^{2}+K_{\beta 3}\left(\beta_{3} / \Delta z\right)^{2}
\end{aligned}
$$

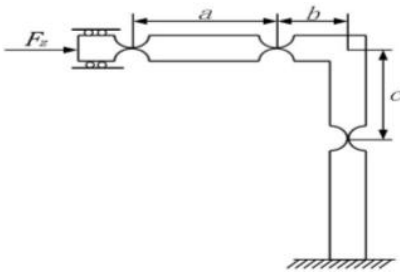

Figure 4(a). The direction diagram of the force $F_{y}$ in the chain

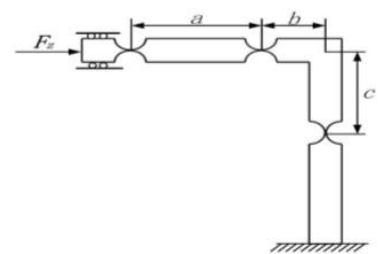

Figure 5(a). The direction diagram of the force $F_{z}$ in the chain

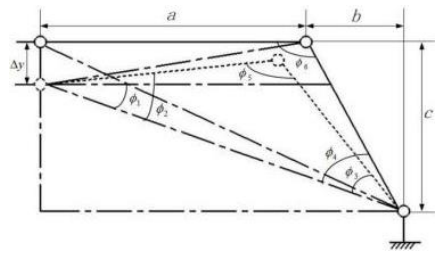

Figure 4(b). The deformable diagram of the force $F_{y}$ in the chain

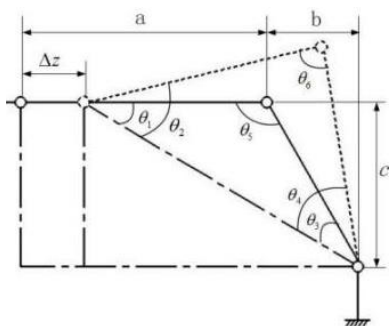

Figure 5(b). The deformable diagram of the force $F_{z}$ in the chain

Stiffness of three chains in the scaning probe are measured:

$\left[\begin{array}{l}k_{1} \\ k_{2} \\ k_{3}\end{array}\right]=\left[\begin{array}{lll}k_{1 x} & k_{1 y} & k_{1 z} \\ k_{2 x} & k_{2 y} & k_{2 z} \\ k_{3 x} & k_{3 y} & k_{3 z}\end{array}\right]$

When the measuring force acting on the measuring probe is $F_{x}$, the distribution of three chains in the space is orthogonal, and the stiffness of the probe in the $\mathrm{X}$ axis is:

$$
\begin{aligned}
& K_{x}=k_{1 x}+k_{2 y}+k_{3 z} \\
& k_{1 x}=\frac{4 K_{1 \alpha}}{l^{2}} \\
& k_{2 y}=K_{\alpha 1}\left(\alpha_{1} / \Delta y\right)^{2}+K_{\alpha 2}\left(\alpha_{2} / \Delta y\right)^{2}+K_{\alpha 3}\left(\alpha_{3} / \Delta y\right)^{2} \\
& k_{3 z}=K_{\beta 1}\left(\beta_{1} / \Delta z\right)^{2}+K_{\beta 2}\left(\beta_{2} / \Delta z\right)^{2}+K_{\beta 3}\left(\beta_{3} / \Delta z\right)^{2}
\end{aligned}
$$

Where, $K_{2 y}$ is subscript 2 represents the branched number, $Y$ is the coordinate of the local coordinate system.

Similarly, when the measuring force are $F_{y}$ and $F_{z}$, the stiffness in $\mathrm{y}$ and $\mathrm{z}$ axises are respectively:

$$
\begin{aligned}
& K_{y}=k_{1 y}+k_{2 z}+k_{3 x} \\
& K_{z}=k_{1 z}+k_{2 x}+k_{3 y}
\end{aligned}
$$

According to the structure size of three chains are identical and completely symmetrical,

$$
K_{x}=K_{y}=K_{z}
$$


Finite Element Calculation for Stiffness of the Probe. When the force F is exerted in the $\mathrm{X}$ direction with finite element analysis method, as shown in Fig. 6, the actual stiffness of the probe is calculated:

$$
k=\frac{F}{\Delta x}
$$

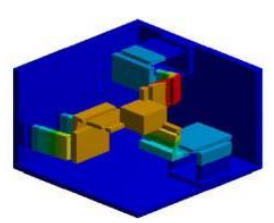

Figure 6. Displacement deformation deformation of scanning probe
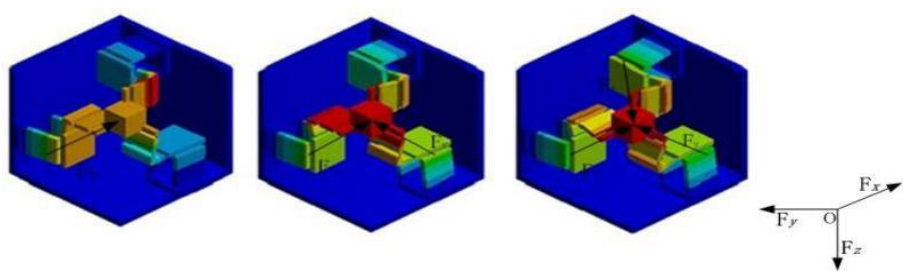

Figure 7. The deformation of the chain in different measuring directions

Where, the span of the hinge in the $X$ axis is 32 millimeters, the span of the hinge(a) is 16.1 millimeters, the span of the hinge(b) is 6 millimeters, the span of the hinge(c) is 14.05 millimeters, the elastic modulus is $7.1 \times 10^{10}$ Pascal, width of the hinge 1 is 1.6 millimeters, width of the hinge 2 is 2.4 millimeters, thickness of the hinge is 0.1 millimeters, radius of the hinge is 1.95 millimeters, the displacement is 0.31648 millimeters. The error of theoretical and the finite element stiffness calculating for the probe are counted to $1.25 \%$.

\section{Error Analysis of Mechanical Structure of the Probe}

In the design, the error is mainly from the difference of the actual movement and the displacement that guiding branched sensor detects. Therefore, minimizing the difference is one of the main methods to solve the error, and also the focus of study.

Error Analysis that the Different Measuring Force Acting on of the Probe. Error Analysis that Measuring Force Acting on in Different Directions. The measuring forces that the size is $\mathrm{F}$ respectively are acted on the direction of $\mathrm{X}$, two directions of $\mathrm{X}, \mathrm{Y}$, three directions of $\mathrm{X}, \mathrm{Y}, \mathrm{Z}$, analyzing the error with finite element analysis and examining Whether 3D decoupling scanning satisfies small mechanical coupling error or not. Fig. 7 show the deformations of each chain after applying the measuring force in different directions to the measuring probe.

In order to verify the decoupling characteristics, after applying measuring forces in different directions to the probe, the error is calculated by the formula: $\Delta x=\left|x_{0}-x_{1}\right|, \Delta x=\left|x_{0}-x_{1}\right|$ $\Delta x=\left|x_{0}-x_{1}\right|, \Delta y=\left|y_{0}-y_{2}\right|, \Delta z=\left|z_{0}-z_{3}\right|$.

Where, $x_{0}$ is the displacement of the probe in $\mathrm{X}$ axis, $y_{0}$ is the displacement of the probe in $\mathrm{Y}$ axis, $z_{0}$ is the displacement of the probe in $\mathrm{Z}$ axis, $x_{1}$ is the displacement that the sensor 1 of the probe detects, $y_{2}$ is the displacement that the sensor 2 of the probe detects, $z_{3}$ is the displacement that the sensor 3 of the probe detects. 
Table 1 The error table of the measuring probe in different measuring directions

\begin{tabular}{|c|c|c|c|c|}
\hline $\begin{array}{l}\text { Measuring } \\
\text { force } \\
\text { direction }\end{array}$ & $\begin{array}{l}\text { Measuri } \\
\text { ng } \\
\text { force/[N] }\end{array}$ & $\begin{array}{c}\text { Measuring } \\
\text { displacement } /[u \\
m] \\
\left(x_{0}, y_{0}, z_{0}\right)\end{array}$ & $\begin{array}{c}\text { Sensor detection } \\
\text { value } /[u m] \\
\left(x_{1}, y_{2}, z_{3}\right)\end{array}$ & $\begin{array}{l}\text { Mechanical } \\
\text { error/[um] }\end{array}$ \\
\hline$x$ & 0.2 & $\begin{array}{c}\text { (316.79、79.741、 } \\
79.762)\end{array}$ & $(316.48,79.665,79.645)$ & $(0.31 、 0.076 、 0.117)$ \\
\hline$x, y$ & 0.2 & $\begin{array}{c}(237.03 、 237.05 、 \\
159.5)\end{array}$ & $(236.83 、 236.81,159.31)$ & $(0.2 、 0.24,0.19)$ \\
\hline$x, y, z$ & 0.2 & $\begin{array}{c}(157.29 、 157.29 、 \\
157.29)\end{array}$ & $(157.17 、 157.17 、 157.17)$ & $(0.12 、 0.12 、 0.12)$ \\
\hline
\end{tabular}

From the analysis of above calculation, can we find when measuring force is acted on the $\mathrm{X}$ axis, the mechanical coupling error of the other two axes is different because of the different stiffness of $\mathrm{Y}$ and $\mathrm{Z}$ axes. With the measuring force acted on multiple axes, the mechanical coupling error reduces gradually because of the symmetrical distribution of the chains. The probe still exists mechanical coupling error when measuring forces are acted on three symmetric directions, because the flexible hinge of the guiding mechanism in force, not only occurs bending deformation but certain torsional deformation. It is a key factor for solving the error to improve the stiffness.

Error Analysis of Measuring Force in Different Sizes. In order to analyze mechanical coupling errors of each guiding mechanisms when the measuring force is acted on single direction. Measuring forces $F_{i}$ of different size are acted on $\mathrm{X}$ axis, the mechanical coupling errors of each guiding chains as show Fig. 8.

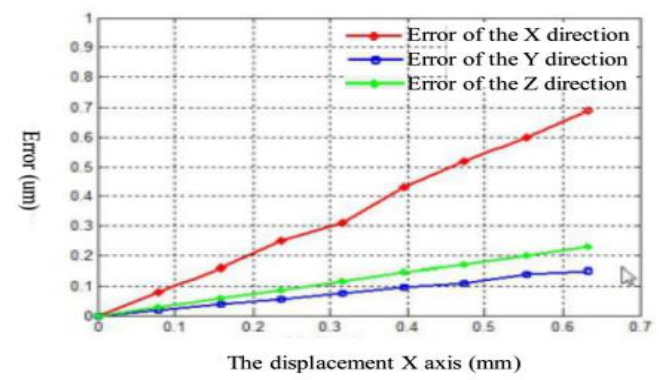

Figure 8 . Analysis diagram of measuring error in single direction of the probe

From the analysis of above diagram, can we find when measuring forces $F_{i}$ are acted on single direction, the absolute errors of each guiding chains gradually increase, but the relative errors are essentially constant in all directions.

Error Analysis of Three-Branch Scanning Probe and Serial Mechanism Probe. The scanning probe with serial mechanism is one of the most common device. Choosing the structure size with parallelogram guiding mechanism of three-branch parallel scanning probe as an example in the design, the mechanical coupling error of three-branch parallel probe and the serical mechanism probe are analyzed respectively. Mechanical coupling error in single axis of common series mechanism probe can be simplified as the mechanical coupling error of a parallelogram flexible hinge. As shown in Fig. 9, the formula of the mechanical coupling error with serical mechanism can be get. When measuring forces $F_{i}$ of the same direction and the different size are acted respectively on parallelogram flexible hinge and three-branch parallel probe, displacement $\Delta$ 
can be get as show in Fig. 10 .

$$
\delta=l-\sqrt{l^{2}-\Delta^{2}}
$$

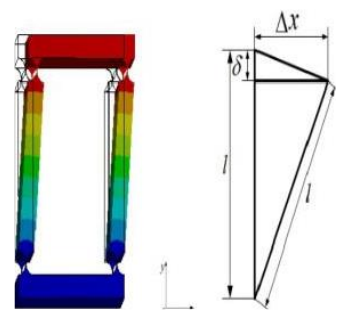

Figure 9. Mechanical coupling error diagram of flexible hinge

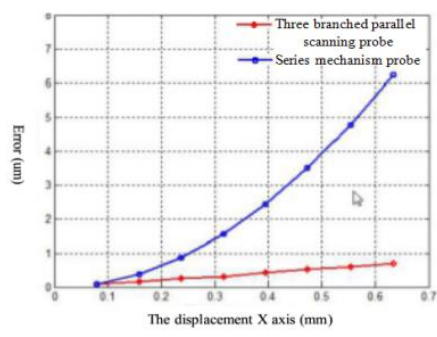

Figure 10. Error Analysis diagram of scanning probe

From the analysis of the error result about three-branch parallel scanning probe and traditional serial mechanism scanning probe, can we find, in certain measuring range, with the increase of displacement, the error of three-branch parallel scanning probe is far less than the serial mechanism probe. At the same time, the relative error of serial probe increases gradually, but the relative error of parallel probe staies unchanged.

\section{Conclusion}

From the analysis of the theoretical stiffness, the finite element simulating stiffness for three-branch parallel probe and the mechanical coupling error, can the conclusions be drawn:

(1) The error of the theoretical stiffness of three-branch parallel probe and the finite element simulating stiffness is less than $2 \%$.

(2) The mechanical coupling error of the scanning probe with parallel structure is much smaller than the scanning probe with traditional serial structure in the design.

(3)The actual displacement of the scanning probe is different from parallelogram guiding mechanism of the chain in the design. This is due to the chain exists not only bending deformation, but also the torsional deformation in measuring.

\section{Acknowledgements}

This work is supported by Shanxi Science and Technology Department of China, with the supported number No. 2016GY-067.

\section{References}

[1] Z.Y. Shi, C.H. Wei. Evolution and some trends in precision probe technology[J].Tool Engineering, 2007,41 (2): 3-8.(In Chinese)

[2] Ottoklingler, kurt. Sensing Head for a Measuring machine: Germany, US4660296 [P]. 1987-4-28.

[3] Fritz Ertrl,Schweinfurt. Multiple-coordinate measurement sensor: Germany, US5029398 [P].1989-11-28.

[4] Mies, Georg. Universal scanning head for gear measurements: Germany, US6131300 [P].2000-10-17.

[5] Meli F,Kung A, Thalmann R.Ultra precision micro-CMM using a low force 3D touch probe. Proc.of.SPIE.2005.

[6] Y.H. Xue. Basic research on parallel numerical control test bench with three degrees of freedom 
based on DELTA mechanism [D]. Hebei University of Technology, 2010.(In Chinese)

[7] Herve J M,Sparacino F.Structure Synthesis of Parallel Robots Generating Spatial Translation [J].5th Int.conf.on Adv.Robotics,IEEE,1991,367-4(1):808-813.

[8] Carricato M,Castelli V P.A Family of 3-DOF Translational Parallel Manipulators [J].Journal of Mechanical Design,2003,125(1):302-307.

[9] Kim H S,Tsai L W.Design optimization of a Cartesian parallel Manipulators[J].Journal of Mechanical Design,2003,125(1):43-51.

[10]F. Gao.Performance analysis and research of 3-DOF translation orthogonal parallel robot[D]. Yanshan University, 2010.(In Chinese)

[11]PAROS J M, WEISBERG L.How to design flexure hinge[J].Machine Design,1965,37(27):151. 